\title{
Ausentismo, presentismo y rendimiento académico en estudiantes de universidades peruanas
}

\section{Absenteeism, Presentism and Academic Performance in Students from Peruvian Universities}

\author{
Raquel Chafloque Céspedes* iD Universidad de San Martín de Porres, Lima, Perú. \\ ORCID: https://orcid.org/0000-0003-0314-5603
}

Arístides Vara-Horna Universidad de San Martín de Porres, Lima, Perú. ORCID: https://orcid.org/0000-0002-3107-1157

Dennis Lopez-Odar (iD) Universidad de San Martín de Porres, Lima, Perú.

ORCID: https://orcid.org/0000-0001-7622-7066

Inés Santi-Huaranca Universidad de San Martín de Porres, Lima, Perú. ORCID: https://orcid.org/0000-0002-4656-5731

Alberto Diaz-Rosillo Universidad de San Martín de Porres, Lima, Perú. ORCID: https://orcid.org/0000-0001-6534-1680

Zaida Asencios-Gonzalez Universidad de San Martín de Porres, Lima, Perú. ORCID: http://orcid.org/0000-0003-0254-8208

Recibido 25-08-17 Revisado 12-09-17Aprobado 30-11-17 En línea 12-12-17

\begin{tabular}{lll} 
*Correspondencia & & Citar como: \\
\cline { 1 - 3 } Email: mchafloquec@usmp.pe & & Chafloque Céspedes, R., Vara-Horna, A., Lopez- \\
& Odar, D., Santi-Huaranca, I., Diaz-Rosillo, A., \\
& \& Asencios-Gonzalez, Z. (2018). Ausentismo, \\
& $\begin{array}{l}\text { presentismo y rendimiento académico en } \\
\text { estudiantes de universidades peruanas. Propósitos }\end{array}$ \\
& $\begin{array}{l}\text { yepresentaciones, 6(1), 83-133. doi: http:// } \\
\text { dx.doi.org/10.20511/pyr2018.v6n1.177 }\end{array}$
\end{tabular}

(C) Universidad San Ignacio de Loyola, Vicerrectorado de Investigación, 2018

(cc) BY-NC-ND Este artículo se distribuye bajo licencia CC BY-NC-ND 4.0 Internacional (http://creativecommons.org/licenses/by-nc-nd/4.0/). 


\section{Resumen}

El objetivo de la presente investigación fue determinar el efecto del ausentismo y presentismo sobre el rendimiento académico en los estudiantes de pregrado de las Escuelas de Negocios e Ingenierías en las Universidades Peruanas. El estudio se sustenta en una encuesta de autoreporte a 8,203 estudiantes de las escuelas de negocios e ingenierías de las universidades peruanas. Se emplearon escalas para analizar el ausentismo, presentismo y rendimiento académico que mostraron adecuadas propiedades psicométricas. Los resultados evidenciaron que el presentismo tiene un mayor impacto sobre el rendimiento académico disminuido de los estudiantes $(\beta=0.628)$ en comparación al ausentismo $(\beta=0.101)$. El ausentismo tiene un mayor efecto sobre los cursos y ciclos desaprobados $(\beta=0.163)$ y el presentismo sobre la evolución del rendimiento académico $(\beta=-0.145)$. Finalmente, mediante un modelo de ecuaciones estructurales se demuestra la estructura causal de la disminución del rendimiento académico a partir del ausentismo y presentismo.

Palabras clave: Ausentismo universitario, presentismo académico, rendimiento académico, Perú.

\section{Summary}

The objective of this research was to determine the effect of absenteeism and presenteeism on the academic performance of undergraduate students from the Business and Engineering Schools in Peruvian Universities. The study is based on a self-report survey of 8,203 students from the business and engineering schools of Peruvian universities. Scales were used to analyze absenteeism, presenteeism and academic performance that showed suitable psychometric properties. The results showed that presenteeism has a higher impact on the academic performance of students $(\beta=0.628)$ compared to absenteeism $(\beta=0.101)$. Absenteeism has a greater effect on courses and disapproved terms $($ Beta $=0.163)$ and presenteeism, on the evolution of 
academic performance $(\beta=-0.145)$. Finally, by means of a model of structural equations, the causal structure of the academic performance decrease from absenteeism and presenteeism is proven.

Keywords: Academic absenteeism, academic presenteeism, academic performance, Peru. 


\section{Introducción}

Del 2005 al 2014 la tasa de matrícula de estudiantes universitarios entre los 20 y 24 años aumentó del $29 \%$ al 33\% a nivel mundial. Asimismo, de los estudiantes de pregrado que estudian a tiempo completo solo el $41 \%$ se gradúan dentro del plazo establecido por el plan de estudio de la carrera profesional (OECD, 2016). Uno de los problemas que influye a que los estudiantes no culminen su formación en este plazo es el ausentismo, el cual constituye un problema creciente en diferentes países (Wadesango \& Machingambi, 2011). Existen evidencias que indican las causas, efectos e implicaciones del ausentismo en los estudiantes, entendido como la acción voluntaria o involuntaria de ausentarse del medio físico del aula, clase o sesión de aprendizaje; determinada por factores académicos y extra académicos (Chilca, 2017; Triado, Aparicio-Chueca, Guárdia-Olmos, JaríaChacón, Cebollero \& Ejjaberi, 2013).

El estudio realizado por Gul et al. (2016) en una muestra de estudiantes de pregrado de la facultad de medicina de una universidad pública, mostró que la prevalencia de ausentismo fue de $87.4 \%$. También, Alghamdi et al. (2016) analizaron la prevalencia del ausentismo en 450 estudiantes, en dos momentos del ciclo de estudios. Sus hallazgos indicaron que la prevalencia antes de los exámenes de medio ciclo fue de $77.6 \%$, mientras que al final de ciclo fue de $70.2 \%$.

El ausentismo en los estudiantes ha sido asociado al rendimiento académico. Diversos estudios han encontrado la relación inversa que existe entre estas variables. Strand \& Granlund (2014), encontró que los estudiantes que empezaban a ausentarse de clases eran los que presentaban mayor dificultad en entender el curso de matemáticas y disminuía su rendimiento académico en el curso. Asimismo, el estudio de Hidayat, Vansal, Kim, Sullivan, \& Salbu, (2012), indica que existe una asociación negativa entre el rendimiento académico y las horas de ausencia en sus cursos. El estudio también indica que los estudiantes de bajo rendimiento reportaron más 
ausencias que los que tenían alto rendimiento. En la misma línea Alghamdi et al. (2016), realizaron su investigación utilizando los promedios de horas perdidas y los rendimientos académicos promedio de los estudiantes, es así que encontraron que las horas ausentes de los estudiantes había impactado negativamente en su rendimiento académico.

Los primeros estudios realizados sobre el concepto de presentismo consideraron al presentismo como una alternativa favorable para reducir el ausentismo en las empresas, ya que los trabajadores asistían a laborar (Uris, 1955; Canfield \& Soash, 1955). Sin embargo, recientemente se ha considerado al presentismo como el antónimo de ausentismo (Janssens, Clays, De Clercq, De Bacquer \& Braeckman, 2013). Otras investigaciones sobre presentismo asocian el concepto a la pérdida de productividad resultante de los trabajadores que asisten a trabajar a pesar de no sentirse bien (BraakmanJansen, Taal, Kuper \& Van de Laar, 2011; Lerner et al., 2012).

Las investigaciones antes mencionadas se basan en estudios realizados en el sector laboral; sin embargo, existen investigaciones que han analizado como se manifiesta el presentismo en los estudiantes (Matsushita et al., 2011; Ferrito, 2016). Son escasas las investigaciones que han conceptualizado el presentismo académico. Los estudios realizados hasta el momento miden y relacionan esta variable con problemas de salud (Deroma, Leach \& Leverett, 2009; Mikami et al., 2013; Matsushita, Yamamura \& Ikeda, 2015) y con la percepción del estudiante en su entorno académico (Ferritto, 2016).

Hysenbegasi, Hass \& Rowland (2005), investigaron la influencia de la depresión en el rendimiento académico, en este estudio el rendimiento académico fue conceptualizado utilizando presentismo relacionado con la depresión y la percepción subjetiva de ausentismo relacionado con la depresión, así como GPA y resultados de la evaluación. En la misma línea, Mikami et al. (2013), encontró que el sentido de coherencia (enfrentar situaciones de estrés) estaba asociado negativamente con el presentismo, lo cual indicaba que el sentido de coherencia disminuía el impacto negativo de los problemas de salud en el rendimiento del estudiante. Por otro lado, 
Ferritto (2016), estudio el presentismo en universitarios basándose en el comportamiento de los estudiantes (prestar atención, la participación, la asistencia, tardanzas y la toma de notas). El estudio brinda información cualitativa que demuestra la relación del presentismo y el rendimiento académico.

La explicación y la investigación de los factores relacionados con el rendimiento académico son temas de suma importancia en la educación superior (Ruban \& McCoach, 2005). En este ámbito se han realizado investigaciones que tienen como objetivo analizar los factores asociados al rendimiento académico del estudiante, asimismo buscan establecer la relación de las variables personales, motivacionales, socioculturales, actitudinales y académicas (Mckenzie \& Schweitzer, 2001; Feldman et al., 2008; Butcher \& Visher, 2013; Busch et al., 2014). En este sentido, la academia no solo busca asociar factores al rendimiento académico, sino identificar los factores con mayor valor predictivo.

Uno de los factores que ha sido relacionado en mayor magnitud al rendimiento académico es el ausentismo, (Alghamdi et al., 2016; Fayombo, Ogunkola \& Olaleye, 2012; Sahim, Arseven \& Kiliç, 2016; Teixeira, 2016). La mayoría de investigaciones que estudian al ausentismo y presentismo como factores asociados a la disminución del rendimiento académico en las universidades, sin embargo, han sido estudiadas individualmente (LópezBonilla \& López-Bonilla, 2015; Alghamdi et al., 2016; Teixeira, 2016; Ferritto, 2016). Las investigaciones que estudian los factores asociados a la disminución del rendimiento académico han empleado muestras pequeñas para sus estudios (Barlow \& Fleischer, 2011; Fayombo et al., 2012; LópezBonilla \& López-Bonilla, 2015; Alghamdi et al., 2016; Sahim et al., 2016), aún no existen muestras nacionales, que muestren la magnitud del problema en conjunto. Incluso aún no se consideran variables como tipo de universidad (pública o privada), escuela (negocios e ingenierías) y tiempo de permanencia de la carrera profesional. 
Las investigaciones realizadas aún no han estudiado al ausentismo y el presentismo como factor asociado a la disminución del rendimiento académico. Sin embargo, cuando el ausentismo ha sido asociado al rendimiento académico se ha demostrado la relación negativa entre estas variables (López-Bonilla \& López-Bonilla, 2015), lo mismo sucede con la asociación del presentismo y rendimiento académico. A diferencia del ausentismo, el presentismo en las universidades es un tema nuevo que aborda la literatura académica.

Frente a este vacío de información, el objetivo del estudio es examinar el ausentismo y el presentismo académico como factores asociados y determinantes de la disminución del rendimiento académico en una muestra nacional y representativa de estudiantes de las carreras de negocios e ingeniería de 34 universidades peruanas.

El conocimiento generado a través de la presente investigación permitirá establecer el valor predictivo del ausentismo y presentismo con relación al rendimiento académico; así como comprender con mayor precisión las implicancias académicas de ambos factores en el contexto universitario peruano. A partir de la evidencia generada, se podrá comprometer a los gestores de los procesos académicos y de formación profesional en la implementación de acciones de orientación, tutoría y seguimiento a los estudiantes; con la finalidad de reducir las tasas de ausentismo, presentismo, rendimiento académico disminuido, cursos y ciclos desaprobados.

\section{Método}

\section{Diseño de investigación.}

El diseño de investigación que se utilizó fue exploratorio, no experimental 


\section{Participantes.}

Participaron 8,203 estudiantes de 34 universidades públicas y privadas de 23 (92.8\%) regiones del Perú. 4,173 mujeres (50.8\%) con una media de edad de 20.43 años (D.E.=1.79) y 4,030 hombres $(49.2 \%$ ) con una media de edad de 20.68 años (D.E.=1.92). Los estudiantes encuestados pertenecían a las escuelas de negocios e ingenierías, del primer al décimo ciclo, el periodo de estudio fue durante el semestre académico 2015-2. En la Tabla 1 se puede observar las características demográficas, académicas y laborales de los estudiantes encuestados.

\section{Tabla 1.}

Características demográficas, académicas y laborales de la muestra (porcentajes).

\begin{tabular}{ll}
\hline Mujeres (4,173) & Hombres (4,030) \\
\hline Año de Ingreso a la Universidad & Año de Ingreso a la Universidad \\
Promedio $=2012$ (D.E. $=1.59)$ & Promedio $=2012($ D.E. $=1.72)$ \\
Rango: $2001-2015$ & Rango: $2000-2015$ \\
Área de Conocimiento & Área de Conocimiento \\
Negocios $=81.5 \%$ & Negocios $=76.4 \%$ \\
Ingenierías $=18.5 \%$ & Ingenierías $=23.6 \%$ \\
Ciclo de universidad & Ciclo de universidad \\
Promedio $=5.47$ ciclos (D.E.=2.67) & Promedio $=5.18$ ciclos (D.E. $=2.74)$ \\
Rango: $1-10$ ciclo & Rango: $1-10$ ciclo \\
Ocupación & Ocupación \\
Solo estudia $=64.0 \%$ & Solo estudia $=52.9 \%$ \\
Estudia y Trabaja $=36.0 \%$ & Estudia y Trabaja $=47.1 \%$ \\
Tiene pareja & Tiene pareja \\
Sí $=48.0 \%$ & Sí $=42.9 \%$ \\
No $=52.0 \%$ & No $=57.1 \%$ \\
\hline
\end{tabular}




\section{Instrumentos.}

El estudio utilizó un cuestionario estructurado bajo el formato de autoreporte, el cual contiene preguntas de información demográfica, académica y laboral, así como las escalas de ausentismo y la escala de presentismo.

Escala de ausentismo: Se incluyeron cinco ítems de la escala utilizada por Reeves \& O'Leary-Kelly (2007). La escala se divide en dos dimensiones, las cuales miden la cantidad de días ausentes por salud y por otras razones. Los ítems tienen alternativas de respuesta ordinales con valores intercalares de interpretación: nunca (0); 1 día (1); 2 días (2); De 3 a 5 días (4); De 6 a 10 días (8); Más de 10 días (15).

Escala de presentismo: Se basa en los ítems de distracción laboral de Stewart, Ricci, Chee, Hann \& Morganstein (2003). Los ítems indican la disminución de productividad académica por distracción y agotamiento. Los ítems tienen alternativas de respuestas ordinales con valores de interpretación intervalar: nunca (0), 1 día (1), entre 2 y 5 días (4), entre 6 y 10 días (8), casi todos los días (15).

Rendimiento académico disminuido: Los ítems permiten medir la disminución percibida del rendimiento académico. Los ítems tienen alternativas de respuestas ordinales con valores de interpretación intervalar: nunca (0), 1 día (1), entre 2 y 5 días (4), entre 6 y 10 días (8), casi todos los días (15). Esta escala se basó en los ítems de distracción laboral (Stewart et al., 2003).

Cursos y ciclos desaprobados: Se utilizaron dos ítems que indicaban la cantidad de ciclos y cursos desaprobados a lo largo de sus estudios.

Evolución del rendimiento académico: Se utilizó un ítem el cual preguntaba al estudiante de cómo calificaba su rendimiento académico actual comparado con el semestre anterior. Tiene 7 alternativas de respuesta "Ha empeorado completamente", "Ha empeorado mucho", "Ha empeorado 
algo", "Se mantiene igual", "Ha mejorado algo", "Ha mejorado mucho" y "Ha mejorado completamente". Asimismo,

\section{Análisis Estadístico.}

Los datos obtenidos fueron analizados con los programas estadísticos SPSS versión 23 y Smart PLS versión 3.2. Para obtener las estadísticas descriptivas se utilizaron tablas de contingencias, medias y porcentajes, con el fin de resumir los datos cuantitativos y estimar las estimaciones de prevalencia de las variables estudiadas. La relación causal de las variables se halló mediante el uso de ecuaciones estructurales de varianza con mínimos cuadrados parciales (SEM - PLS), se analizó la relación causal entre las escalas. Se ha utilizado el programa estadístico SmartPLS, para calcular la significación de las relaciones entre los constructos (Ringle, Wende \& Becker, 2015). Para calcular el impacto del ausentismo y presentismo en el rendimiento académico se utilizaron los algoritmos, descritos en cada escala.

\section{Validez y fiabilidad.}

Para el análisis de fiabilidad de cada escala, se usó el coeficiente de confiabilidad del Alfa de Cronbach. La escala de ausentismo ( $\alpha=0.692)$, obtuvo un Alfa inferior al mínimo esperado $(\alpha=0.706)$ esto se debe a que la escala presenta datos excluyentes, esto no indica algún problema ya que las razones de ausentismo a la universidad son diferentes y esta puede excluir a la otra. La escala de presentismo $(\alpha=0.794)$ obtuvo un valor Alfa superior al mínimo esperado, mientras que la escala de rendimiento académico disminuido $(\alpha=0.606)$.

En cuanto al análisis de validez de constructo se realizó por medio del Análisis de Componentes Principales (ACP) con rotación Varimax. Los 13 ítems en estudio ( 5 de la escala de ausentismo, 4 de la escala de presentismo, 2 de rendimiento académico disminuido y 2 de cursos y ciclos desaprobados), 
proporcionaron una solución factorial de 3 componentes con una varianza total de $57.2 \%$ de los datos originales (ver Tabla 2).

\section{Tabla 2.}

Validez factorial de los items de las escalas de ausentismo, presentismo y rendimiento académico, mediante el ACP con rotación Varimax.

\begin{tabular}{|c|c|c|c|c|}
\hline \multirow{2}{*}{ Ítems } & \multicolumn{3}{|c|}{ Factores } & \multirow{2}{*}{ Comunalidades } \\
\hline & 1 & 2 & 3 & \\
\hline $\begin{array}{l}\text { Tuvo dificultades para concentrarse } \\
\text { estuvo distraído en clases o } \\
\text { mientras estudiaba. }\end{array}$ & .828 & & & 692 \\
\hline Estudió más lento de lo usual & .803 & & & .649 \\
\hline $\begin{array}{l}\text { Estuvo cansado, agotado o exhausto } \\
\text { mientras estaba en clases. }\end{array}$ & .790 & & & .630 \\
\hline $\begin{array}{l}\text { Tuvo preocupaciones personales } \\
\text { ajenas al estudio }\end{array}$ & .707 & & & .529 \\
\hline $\begin{array}{l}\text { Cometió errores en sus exámenes } \\
\text { o presentación de trabajos porque } \\
\text { estaba preocupado o algo le } \\
\text { afectaba }\end{array}$ & .842 & & & .707 \\
\hline $\begin{array}{l}\text { Tuvo un rendimiento académico } \\
\text { muy por debajo de sus capacidades }\end{array}$ & .847 & & & .717 \\
\hline $\begin{array}{l}\text { Para atender su salud física o } \\
\text { mental. }\end{array}$ & & .750 & & .568 \\
\hline $\begin{array}{l}\text { Para atender la salud o cuidado } \\
\text { de otros familiares o personas } \\
\text { cercanas. }\end{array}$ & & .710 & & .506 \\
\hline Por estar enfermo o indispuesto. & & .704 & & .513 \\
\hline $\begin{array}{l}\text { Para atender temas personales, } \\
\text { legales o financieros. }\end{array}$ & & .644 & & .426 \\
\hline Por otras razones. & & .502 & & .288 \\
\hline $\begin{array}{l}\text { Ciclos repetidos a lo largo de sus } \\
\text { estudios universitarios. }\end{array}$ & & & .863 & .749 \\
\hline $\begin{array}{l}\text { Cursos desaprobados a lo largo de } \\
\text { sus estudios universitarios. }\end{array}$ & & & .851 & .742 \\
\hline
\end{tabular}

La escala de presentismo y rendimiento académico disminuido saturo completamente el primer factor con pesos factoriales entre $0.707 \mathrm{y}$ 
0.847, los 6 ítems cumplieron los supuestos para ser validados ya que los pesos factoriales superaron el mínimo esperado. Asimismo, la escala de ausentismo saturo el segundo factor con sus 5 ítems, sin embargo 2 de sus pesos factoriales se encuentran por debajo del mínimo esperado $(\alpha=0.706)$ y comunalidades menores al $50 \%$. Por último, los ítems de cursos y ciclos desaprobados superaron los supuestos para ser validados, con pesos de 0.863 y 0.851 .

\section{Modelo estructural.}

Mediante el uso de las ecuaciones estructurales de varianza de mínimos cuadrados parciales (SEM-PLS), se analizó la validez de constructo, así como también se analizó la fiabilidad compuesta. Todos los pesos factoriales de los ítems de las 3 escalas fueron superiores al mínimo esperado (0.706). Las escalas de presentismo, ausentismo y rendimiento académico obtuvieron altos niveles de fiabilidad compuesta $0.865,0.838$ y 0.784 y con varianza extraída promedio por escala entre el 0.569 y el 0.712 . 


\section{Tabla 3.}

Validez de constructo de los items de las escalas de ausentismo, presentismo y rendimiento académico, mediante Ecuaciones Estructurales de Varianza usando Minimos Cuadrados Parciales.

\begin{tabular}{|c|c|c|c|}
\hline Ítems & $\begin{array}{c}\text { Peso } \\
\text { Factorial }\end{array}$ & $\begin{array}{l}\text { Fiabilidad } \\
\text { compuesta }\end{array}$ & $\begin{array}{l}\text { Varianza } \\
\text { extraída }\end{array}$ \\
\hline $\begin{array}{l}\text { Tuvo dificultades para concentrarse estuvo } \\
\text { distraído en clases o mientras estudiaba. }\end{array}$ & 0.810 & \multirow{4}{*}{0.865} & \multirow{4}{*}{0.421} \\
\hline Estudió más lento de lo usual & 0.800 & & \\
\hline $\begin{array}{l}\text { Estuvo cansado, agotado o exhausto } \\
\text { mientras estaba en clases. }\end{array}$ & 0.787 & & \\
\hline $\begin{array}{l}\text { Tuvo preocupaciones personales ajenas al } \\
\text { estudio }\end{array}$ & 0.740 & & \\
\hline Para atender su salud física o mental. & 0.714 & \multirow{5}{*}{0.784} & \multirow{5}{*}{0.616} \\
\hline $\begin{array}{l}\text { Para atender la salud o cuidado de otros } \\
\text { familiares o personas cercanas. }\end{array}$ & 0.682 & & \\
\hline Por estar enfermo o indispuesto. & 0.604 & & \\
\hline $\begin{array}{l}\text { Para atender temas personales, legales o } \\
\text { financieros. }\end{array}$ & 0.632 & & \\
\hline Por otras razones. & 0.606 & & \\
\hline $\begin{array}{l}\text { Cometió errores en sus exámenes o } \\
\text { presentación de trabajos porque estaba } \\
\text { preocupado o algo le afectaba }\end{array}$ & 0.841 & \multirow[t]{2}{*}{0.838} & \multirow[t]{2}{*}{0.722} \\
\hline $\begin{array}{l}\text { Tuvo un rendimiento académico muy por } \\
\text { debajo de sus capacidades }\end{array}$ & 0.858 & & \\
\hline $\begin{array}{l}\text { Ciclos repetidos a lo largo de sus estudios } \\
\text { universitarios. }\end{array}$ & 0.814 & \multirow[b]{2}{*}{0.850} & \multirow[b]{2}{*}{0.739} \\
\hline $\begin{array}{l}\text { Cursos desaprobados a lo largo de sus } \\
\text { estudios universitarios. }\end{array}$ & 0.904 & & \\
\hline $\begin{array}{l}\text { Percepción de la evolución del rendimiento } \\
\text { académico. }\end{array}$ & 1.000 & 1.000 & 1.000 \\
\hline
\end{tabular}

Se utilizó el criterio de Fornell - Larcker, para analizar la validez discriminante, donde el promedio de varianza extraída (AVE) ha de ser mayor que las correlaciones que presentan con el resto de dimensiones. En 
la tabla 4 se observa que se cumple con ese criterio en todas las subescalas (diagonales entre paréntesis) demostrando validez discriminante.

\section{Tabla 4.}

Validez discriminante de las escalas de ausentismo, presentismo y rendimiento académico.

\begin{tabular}{lccccc}
\hline & Ausentismo & $\begin{array}{c}\text { Cursos } \\
\text { y ciclos } \\
\text { desaprobados }\end{array}$ & $\begin{array}{c}\text { Evolución } \\
\text { rendimiento } \\
\text { académico }\end{array}$ & Presentismo & $\begin{array}{c}\text { Rendimiento } \\
\text { académico } \\
\text { disminuido }\end{array}$ \\
\hline $\begin{array}{l}\text { Ausentismo } \\
\begin{array}{l}\text { Cursos } \\
\text { y ciclos } \\
\text { desaprobados }\end{array}\end{array}$ & $\mathbf{( 0 . 6 4 9 )}$ & & & & \\
$\begin{array}{l}\text { Evolución de } \\
\text { rendimiento } \\
\text { académico }\end{array}$ & -0.180 & $\mathbf{( 0 . 8 6 0 )}$ & & & \\
$\begin{array}{l}\text { Presentismo } \\
\begin{array}{l}\text { Rendimiento } \\
\text { académico } \\
\text { disminuido }\end{array}\end{array}$ & 0.231 & 0.025 & $\mathbf{( 1 . 0 0 0 )}$ & & \\
\hline
\end{tabular}

\section{Procedimiento}

La encuesta fue administrada y asistida presencialmente por profesionales capacitados. Los estudiantes fueron informados de los objetivos y alcances del estudio, se garantizó la confidencialidad y el anonimato. Los estudiantes dieron consentimiento verbal antes de la recolección de datos, además de también realizar preguntas de consentimiento en la parte final del cuestionario. Su participación fue voluntaria, sin ninguna clase de incentivos académicos, económicos o de otro tipo. Los estudiantes completaron la encuesta durante horas de clase, el tiempo promedio fue de 25 minutos.

\section{Resultados}

El 78.8\% de los estudiantes se ausentaron de clases, al menos un día en el último mes, los estudiantes indicaron que las ausencias se dieron por 
estar enfermo o indispuesto (53\%), por otras razones (45.5\%), para atender la salud o cuidado de otros familiares o personas cercanas $(36.9 \%)$, para atender temas personales, legales o financieras $(35.8 \%)$ y para atender su salud física o mental (34.6\%). El promedio de días de ausentismo al año por cualquiera de estos motivos es de 44.89 días (D.E. $=48.23$ ).

En cuanto a los estudiantes que reportaron presentismo, el $95.7 \%$ de los estudiantes tuvo problemas para concentrarse durante las horas de clase. El $82.3 \%$ de los estudiantes estuvieron distraídos, asimismo indicaron que estuvieron cansados (87.2\%), estudiaron más lento $(77.4 \%)$ y tuvieron preocupaciones personales ajenas al estudio $(76.3 \%)$.

E1 $82.5 \%$ de los estudiantes manifestaron que su rendimiento académico estuvo por debajo de sus capacidades en el último mes, mientras que el $71 \%$ mencionaron que cometieron errores en sus exámenes o trabajos.

E1 53.6\% de los estudiantes indicaron que han desaprobado al menos un curso y el $23 \%$ ha repetido al menos un ciclo, en el transcurso de los ciclos académicos estudiados. Diferenciado por sexo, el 51.8\% de estudiantes mujeres desaprobaron al menos un curso, el promedio de cursos desaprobados fue de 2.64 (D.E.=1.97), mientras que el 55.4\% de estudiantes hombres desaprobaron al menos un curso y se encontró que el promedio de cursos desaprobados por parte de los alumnos fue de 3.13 cursos (D.E.=2.28). Los ciclos repetidos por los estudiantes diferenciado por sexo, el $18.2 \%$ de estudiantes mujeres han repetido un ciclo académico, el promedio de ciclos repetidos es de 1.7 (D.E. $=1.01$ ), en un rango de 1 a 6 ciclos repetidos. Asimismo, el $23.8 \%$ de estudiantes hombres habría repetido al menos un ciclo académico, el promedio de ciclos repetidos fue de 1.76 ciclos (D.E. $=1.05$ ), en un rango de 1 a 7 ciclos repetidos.

\section{Modelo estructural.}

Se analizó la asociación del ausentismo y presentismo académico en el rendimiento académico disminuido, encontrándose que las variables tienen 
un efecto directo en el rendimiento académico disminuido, explicándolo en un $43.4 \%$. En la Figura 1, también se observa que el ausentismo explica el $5.3 \%$ del presentismo académico. Al mismo tiempo, el ausentismo explica el $3.7 \%$ del cursos y ciclos desaprobados. Asimismo, el ausentismo y el presentismo académico explican el $9.7 \%$ de las limitaciones que presentan los estudiantes para mejorar la evolución de su rendimiento académico.

Para corregir la atenuación de las correlaciones entre las escalas y hacerlo equiparable a un modelo de covarianzas, se utiliza el algoritmo Consistent PLS (PLSc), pues con este método se pueden obtener resultados más estables (Dijkstra \& Henseler, 2012).

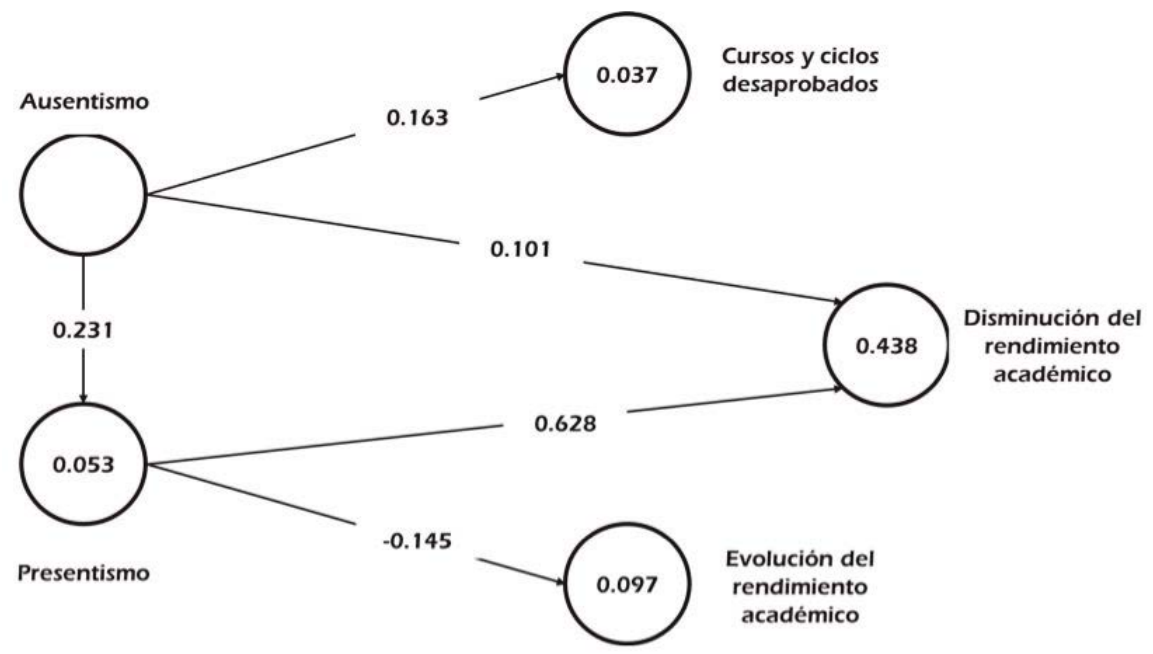

Figura 1. Diagrama Causal del Impacto de Ausentismo y Presentismo en el rendimiento académico.

\section{Modelo de medición.}

En la Tabla 5, se observa la significancia de los coeficientes de trayectoria del modelo. En general todas las relaciones son estables y muy semejantes a los para parámetros poblacionales pues cuando se evaluó el significado de la relación hipotética el valor $t$ fue significativa para todas relaciones propuestas. Sin embargo, la relación de ausentismo y la evolución del 
rendimiento académico no es significativa, lo mismo sucede con la relación del presentismo a los cursos y ciclos desaprobados.

\section{Tabla 5.}

Significancia de los coeficientes de trayectoria (beta) entre el ausentismo, presentismo académico y variables del rendimiento académico.

\begin{tabular}{|c|c|c|c|c|c|}
\hline $\begin{array}{l}\text { Relaciones entre dimensiones } \\
\text { (valores Beta) }\end{array}$ & $\begin{array}{l}\text { Muestra } \\
\text { original } \\
\text { (O) }\end{array}$ & $\begin{array}{l}\text { Promedio } \\
\text { muestral (M) }\end{array}$ & $\begin{array}{c}\text { Error } \\
\text { estándar } \\
\text { (STERR) }\end{array}$ & $\begin{array}{l}\text { T Statistics } \\
(\mathrm{O} / \mathrm{STERR})\end{array}$ & $\mathrm{P}$ \\
\hline $\begin{array}{l}\text { Ausentismo } \rightarrow \text { Cursos y } \\
\text { ciclos desaprobados }\end{array}$ & 0.163 & 0.163 & 0.014 & 11.931 & 0.000 \\
\hline $\begin{array}{l}\text { Ausentismo } \rightarrow \text { Evolución de } \\
\text { rendimiento académico }\end{array}$ & -0.024 & -0.024 & 0.012 & 1.942 & 0.052 \\
\hline Ausentismo $\rightarrow$ Presentismo & 0.231 & 0.231 & 0.012 & 19.195 & 0.000 \\
\hline $\begin{array}{l}\text { Ausentismo } \rightarrow \text { Disminución } \\
\text { de rendimiento académico }\end{array}$ & 0.101 & 0.102 & 0.012 & 8.743 & 0.000 \\
\hline $\begin{array}{l}\text { Presentismo } \rightarrow \text { Cursos y } \\
\text { ciclos desaprobados }\end{array}$ & -0.005 & -0.006 & 0.015 & 0.376 & 0.707 \\
\hline $\begin{array}{l}\text { Presentismo } \rightarrow \text { Evolución de } \\
\text { rendimiento académico }\end{array}$ & -0.145 & -0.145 & 0.015 & 9.979 & 0.000 \\
\hline $\begin{array}{l}\text { Presentismo } \rightarrow \text { Disminución } \\
\text { de rendimiento académico }\end{array}$ & 0.628 & 0.628 & 0.010 & 63.138 & 0.000 \\
\hline
\end{tabular}

Nota: Simulación mediante Bootstraping. Remuestreo (5000 veces). Significativos, $\mathrm{p}<0.01$

Los coeficientes de trayectoria beta han sido significativos, sin embargo, es necesario determinar el tamaño de efecto de cada relación. Tal como se observa en la Tabla 6, la relación con mayor tamaño de efecto es la del presentismo y el rendimiento académico disminuido. A pesar de que existen relaciones con tamaño de efecto muy pequeño, estas resultan significativas, ya que sus intervalos de confianza oscilan entre 0.01 y 0.07 . Al igual que los coeficientes de trayectoria el tamaño de efecto en las relaciones Ausentismo $\rightarrow$ Evolución de rendimiento académico y Presentismo $\rightarrow$ Cursos y ciclos desaprobados, no son significativos. 


\section{Tabla 6.}

Tamaño del efecto de los coeficientes beta entre el ausentismo, presentismo académico y variables del rendimiento académico.

\begin{tabular}{lccccc}
\hline $\begin{array}{c}\text { Relaciones entre dimensiones } \\
\text { (valores Beta) }\end{array}$ & $\mathrm{F}^{2}(\mathrm{O})$ & $\mathrm{F}^{2}(\mathrm{M})$ & $\begin{array}{c}\text { Error estándar } \\
(\mathrm{STERR})\end{array}$ & $\begin{array}{c}\mathrm{T} \\
\text { Statistics }(\mid \mathrm{O} / \\
\mathrm{STERR} \mid)\end{array}$ & $\mathrm{P}$ \\
\hline $\begin{array}{l}\text { Ausentismo } \rightarrow \text { Cursos y ciclos } \\
\text { desaprobados }\end{array}$ & 0.026 & 0.026 & 0.004 & 5.767 & 0.000 \\
$\begin{array}{l}\text { Ausentismo } \rightarrow \text { Evolución de } \\
\text { rendimiento académico }\end{array}$ & 0.001 & 0.001 & 0.001 & 0.900 & 0.368 \\
$\begin{array}{l}\text { Ausentismo } \rightarrow \text { Presentismo } \\
\text { Ausentismo } \rightarrow \text { Disminución de } \\
\text { rendimiento académico }\end{array}$ & 0.056 & 0.057 & 0.006 & 9.028 & 0.000 \\
$\begin{array}{l}\text { Presentismo } \rightarrow \text { Cursos y ciclos } \\
\text { desaprobados }\end{array}$ & 0.000 & 0.000 & 0.000 & 0.0918 & 0.0000 \\
$\begin{array}{l}\text { Presentismo } \rightarrow \text { Evolución de } \\
\text { rendimiento académico }\end{array}$ & 0.013 & 0.014 & 0.003 & 4.920 & 0.000 \\
$\begin{array}{l}\text { Presentismo } \rightarrow \text { Disminución de } \\
\text { rendimiento académico }\end{array}$ & 0.659 & 0.660 & 0.034 & 19.577 & 0.000 \\
\hline
\end{tabular}

Nota: Simulación mediante Bootstraping. Remuestreo (5000 veces). Significativos, $\mathrm{p}<0.01$.

\section{Contrastación de Hipótesis.}

Para probar la hipótesis, que muestra al ausentismo y presentismo académico como factores asociados al rendimiento académico disminuido, se desarrolló un modelo, el cual supone que el ausentismo y el presentismo influyen en la percepción del rendimiento académico disminuido de los estudiantes. Por otra parte, se asume que el ausentismo tiene un efecto directo en el presentismo y ambos afectan la evolución del rendimiento académico. Finalmente, se plantea que el ausentismo tiene un mayor efecto sobre los cursos y ciclos desaprobados.

\section{Discusión}

La disminución del rendimiento académico en las universidades es una preocupación latente debido a las consecuencias sobre el estudiante y la 
sociedad en general. El presente estudio investiga al ausentismo y presentismo como factores asociados a la disminución del rendimiento académico en los estudiantes de pregrado en las escuelas de negocios e ingenierías de las universidades peruanas. Con base a los resultados obtenidos se demuestra que el ausentismo y el presentismo son factores asociados que explican la disminución del rendimiento académico de los estudiantes en un $43.8 \%$. Este resultado se respalda en diversos estudios de la academia los cuales han encontrado que el rendimiento académico es multicausal y complejo, y que la interacción de múltiples factores sociales, personales e institucionales pueden variar de acuerdo a la muestra (Garbanzo, 2013).

El ausentismo reportado en el estudio es $78.8 \%$, este resultado es menor al que reportan Wadesango \& Machingabi (2011), los investigadores indicaron que el $100 \%$ de su muestra se ausento muchas veces durante el ciclo académico, la muestra que utilizaron los autores fue una muestra de 42 participantes, se puede deducir que el tamaño de su muestra puede condicionar la incidencia de ausentismo, la muestra utilizada en la presente investigación es una muestra nacional aleatoria. Por otro lado, el presente estudio analiza el ausentismo ocurrido durante un mes anterior al que se recogen los datos. Las principales causas de ausentismo según lo informado por los estudiantes fueron por estar enfermo o indispuesto y por otras razones, estos resultados se asemejan a estudios anteriores (Barlow \& Fleischer, 2011; Fayombo et al., 2012).

Asimismo, el presentismo académico encontrado en los estudiantes fue de $95.7 \%$, encontrándose que las mujeres tienen más presentismo que los hombres. El presente estudio muestra resultados cuantitativos antes no hallados en una muestra representativa nacional, no obstante, estos datos van acorde con el estudio de Matsushita et al. (2011), donde se encontró que el presentismo en los estudiantes es frecuente, además el estudio indica que las mujeres tienen mayor presentismo que los estudiantes hombres. El presentismo en estudiantes universitarios puede presentarse con mayor frecuencia en el salón de clases y esto puede suceder debido a que el 
estudiante no quiera faltar al salón de clases para evitar inasistencias, sin embargo, el estudiante con presentismo no logrará una concentración plena.

En el presente estudio se ha utilizado las Ecuaciones Estructurales de Varianza con Mínimos Cuadrados Parciales (SEM-PLS), para hallar la asociación del ausentismo y el presentismo en la disminución del rendimiento académico. Estudios previos han encontrado la relación inversa que existe entre el ausentismo y el rendimiento académico, lo mismo sucede al momento que se analiza la variable del presentismo (Alghamdi et al., 2016; Ferritto, 2016). A pesar de los estudios realizados, la academia aún no ha realizado un estudio que analicé al ausentismo y presentismo académico como factores asociados en la disminución del rendimiento académico en universidades peruanas, utilizando el SEM-PLS. En el modelo propuesto, se corrobora que el ausentismo genera un impacto directo en los cursos y ciclos desaprobados, está relación se evidencia con los coeficientes path (mayores a 1.0) y $\mathrm{R}^{2}$ la varianza explicada (mayores a 1.0).

Asimismo el modelo también nos presenta que el ausentismo y el presentismo influyen en la evolución del rendimiento académico, donde el vínculo causal se explica de la siguiente manera: los estudiantes que se han ausentado en algún momento del mes por motivos de salud u otras razones han provocado el presentismo en los estudiantes distrayéndolos o influyendo en la desconcentración de estos durante el horario de clases, esto provoco limitaciones en los estudiantes lo cual afecto de manera negativa la evolución del rendimiento académico. En esta relación, se observa un mayor efecto del ausentismo y presentismo sobre la evolución del rendimiento académico, si se relaciona directamente al ausentismo con la evolución del rendimiento académico, se obtienen tamaños de efecto no significativos.

La técnica que se utiliza para explicar la asociación de las variables, es una técnica analítica que puede ser utilizado para explicar la relación causa y efecto con los modelos conceptuales desarrollados dentro de las investigaciones, tiene un enfoque integral, que va más allá de ANOVA o análisis basado en regresión lineal. Dada la importancia principal de 
este problema social, con SEM-PLS se demuestra que el ausentismo y el presentismo académico genera un rendimiento académico disminuido.

Los principales aportes de la presente investigación, son los resultados estadísticos del ausentismo universitario, del presentismo académico y el rendimiento académico disminuido en una muestra nacional, asimismo brinda información cualitativa sobre el presentismo académico el cual es un tema que recién se está aplicando al entorno universitario, por ultimo explica la asociación del ausentismo y el presentismo basándose en el SEMPLS y como ambas variables se asocian a la disminución del rendimiento académico.

Es importante realizar más investigaciones que expliquen al presentismo como factor asociado a la disminución del rendimiento académico, ya que es un factor que engloba diversas características que se vienen estudiando individualmente ya sea como características de salud o características emocionales. Asimismo, se recomienda que para futuras investigaciones se sigan incluyendo muestras nacionales y se realicen estudios longitudinales.

Finalmente, la evidencia encontrada permite que las entidades públicas y privadas del sector educativo tomen las acciones pertinentes para plantear políticas de estado las cuales intervengan en la disminución del ausentismo y presentismo a nivel nacional. El trabajo conjunto entre estados, universidades y estudiantes es necesario para disminuir un problema latente que si se deja persistir a largo plazo provocará deserción estudiantil.

\section{Referencias}

Alghamdi, A., Yamani, A., Khalil, A., Albarkati, B., Alrehili, O. \& Salih, M. (2016). Prevalence causes and impacts of absenteeism among medical students at UQU. Education, 6(1), 9 -12. Doi: https://10.5923/j. edu. 20160601.02

Barlow, J., \& Fleischer, S. (2011). Student absenteeism: Whose responsibility? Innovations in Education and Teaching International, 48(3), 227-237. Doi: https://doi.org/10.1080/14703297.2011.593700 
Braakman-Jansen, L. M., Taal, E., Kuper, I. H., \& Van de Laar, M. A. (2011). Productivity loss due to absenteeism and presenteeism by different instruments in patients with RA and subjects without RA. Rheumatology, 51(2), 354-361. Doi: https://doi.org/10.1093/rheumatology/ker371

Busch, V., Loyen, A., Lodder, M., Schrijvers, A. J., Van Yperen, T. A., \& De Leeuw, J. R. (2014). The effects of adolescent health-related behavior on academic performance: a systematic review of the longitudinal evidence. Review of Educational Research, 84(2), 245 - 274. Doi: https://doi. org/10.3102/0034654313518441

Butcher, K. F., \& Visher, M. G. (2013). The impact of a classroom-based guidance program on student performance in community college math classes. Educational Evaluation and Policy Analysis, 35(3), 298-323. Doi: https://doi.org/10.3102/0162373713485813

Canfield, G. W., Soash, D. G. (1955). Presenteeism - A constructive view. Personnel Journal 34, 94-97.

Chilca, L. (2017). Autoestima, hábitos de estudio y rendimiento académico en estudiantes universitarios. Propósitos y Representaciones, 5(1), 71127. Doi: https://doi.org/10.20511/pyr2017.v5n1.145

Deroma, V. M., Leach, J. B., \& Leverett, J. P. (2009). The relationship between depression and college academic performance. College Student Journal, 43(2), 325-335.

Dijkstra, T. K. \& Henseler, J. (2012). Consistent and asymptotically normal PLS - estimators for linear structural equations (Workin Paper). Recuperado de: http://www.rug.nl/staff/t.k.dijkstra/research

Fayombo, G., Ogunkola, B., \& Olaleye, Y. (2012). Cross institutional study of the causes of absenteeism among university students in Barbados and Nigeria. Journal of Educational and Developmental Psychology, 2(1), 122. Doi: https://doi.org/10.5539/jedp.v2n1p122

Feldman, L., Goncalves, L., Puignau, G. C. C., Zaragoza, J., Bagés, N., \& De Paulo, J. (2008). Relaciones entre estrés académico, apoyo social, salud mental y rendimiento académico en estudiantes universitarios venezolanos. Universitas psychologica, 7(3), 739-752. 
Ferritto, V. R. (2016). Maritime education factors and presenteeism: A comparative quantitative study. WMU Journal of Maritime Affairs, 15(2), 353-380. Doi: https://doi.org/10.1007/s13437-015-0098-9

Garbanzo (2013). Factores asociados al rendimiento académico en estudianyes universitarios desde el nivel socieconómico. Un studio en la Universidad de Costa Rica. Revista Electrónica Educare. 17(3), 57-87.

Gul, R., Khan, H. M., Alam, S. R., Luqman, F., Shahab, A., \& Sohail, H. (2016). Absenteeism among medical undergraduate students. Journal of Medical Sciences, 24(1), 16 - 18.

Hidayat, L., Vansal, S., Kim, E., Sullivan, M., \& Salbu, R. (2012). Pharmacy student absenteeism and academic performance. American Journal of Pharmaceutical Education, 76(1). Doi: https://doi.org/10.5688/ajpe7618

Hysenbegasi, A., Hass, S., \& Rowland, C. (2005). The impact of depression on the academic productivity of university students. J Men Health Policy and Econ, 8(3), 145-151

Janssens, H., Clays, E., De Clercq, B., De Bacquer, D., \& Braeckman, L. (2013). The relation between presenteeism and different types of future sickness absence. Journal of occupational health, 55(3), 132-141. Doi: https://doi.org/10.1539/joh.12-0164-OA

Lerner, D., Chang, H., Rogers, W., Benson, C., Chow, W., Kim, M., \& Biondi, D. (2012). Imputing at-work productivity loss using results of a randomized controlled trial comparing tapentadol extended release and oxycodone controlled release for osteoarthritis pain. Journal of occupational and environmental medicine, 54(8), 933-938. Doi: https:// doi.org/10.1097/JOM.0b013e31825f31a1

López-Bonilla, J. M., \& López-Bonilla, L. M. (2015). The multidimensional structure of university absenteeism: An exploratory study. Innovations in Education and Teaching International, 52(2), 185-195. Doi: https:// doi.org/10.1080/14703297.2013.847382

McKenzie K., \& Schweitzer R. (2001). Who Succeeds at University? Factors predicting academic performance in first year Australian university students. Higher education research \& development, 20(1), 21-33. Doi: https://doi.org/10.1080/07924360120043621 
Matsushita, M., Adachi, H., Arakida, M., Namura, I., Takahashi, Y., Miyata, M., \& Sugita, Y. (2011). Presenteeism in college students: Reliability and validity of the presenteeism scale for students. Quality of Life Research, 20(3), 439-446. Doi: https://doi.org/10.1007/s11136-010-9763-9

Matsushita, M., Yamamura, S. \& Ikeda, M. (2015) Investigation of Prefrontal Cortex Activity in University Students with Presenteeism: A NearInfrared Spectroscopy (NIRS) Study. Journal of Behavioral and Brain Science, 5, 339-347. Doi: https://doi.org/10.4236/jbbs.2015.59034

Mikami, A., Matsushita, M., Adachi, H., Suganuma, N., Koyama, A., Ichimi, N., \& Sugita, Y. (2013). Sense of coherence, health problems, and presenteeism in japanese university students. Asian Journal of Psychiatry, 6(5), 369-372. Doi: https://doi.org/10.1016/j.ajp.2013.03.008

OECD (2016), Education at a Glance 2016: OECD Indicators. OECD Publishing, Paris. Recuperado de: http://dx.doi.org/10.187/eag-2016-en

Reeves, C., \& O' Leary-Kelly, A. (2009). Study of the effects intimate partner violence on the workplace. Retrieved from https://www.ncjrs. gov/pdffiles1/nij/grants/227266.pdf

Ringle, C. M., Wende, S., \& Becker, J. M. (2015). SmartPLS. Boenningstedt, Germany: SmartPLS GmbH. Recuperado de: from www.smartps.com

Ruban, L. M., \& McCoach, D. B. (2005). Gender differences in explaining grades using structural equation modelling. Review of Higher Education, 28, 475-502. Doi: https://doi.org/10.1353/rhe.2005.0049

Sahim, S., Arseven, Z., \& Kiliç, A. (2016). Causes of student absenteeism and school dropouts. International Journal of Instruction, 9(1), 195-210. Recuperado de: https://eric.ed.gov/?id=EJ1086967

Strand, A. S., \& Granlund M. (2014). The School Situation for Students with a High Level of Absenteeism in Compulsory School: Is There a Pattern in Documented Support? Scandinavian Journal of Educational Research, 58:5, 551-569, Doi: https://doi.org/10.1080/00313831.2013. 773561

Stewart, W. F., Ricci, J. A., Chee, E., Hahn, S. R., \& Morganstein, D. (2003). Cost of lost productive work time among US workers with depression. Journal of the American Medical Association, 289(23), 3135-3144. Doi: https://doi.org/10.1001/jama.289.23.3135 
Teixeira, A. (2016) The impact of class absenteeism on undergraduates' academic performance: evidence from an elite Economics school in Portugal, Innovations in Education and Teaching International, 53:2, 230-242, Doi: https://doi.org/10.1080/14703297.2014.937730

Triado, X. M., Aparicio-Chueca, P., Guàrdia-Olmos, J., Jaría-Chacón, N., Cebollero, M. P., \& Ejjaberi, A. E. (2013). University student absenteeism: Factors and profiles. Handbook of research on transnational higher education, 454-466. Doi: https://doi.org/10.4018/978-1-4666-4458-8. ch023

Uris, A. (1955). How to build presenteeism. Petroleum Refin. 34, 348-359

Wadesango, N., \& Machingambi, S. (2011). Causes and structural effects of student absenteeism: a case study of three South African Universities. Journal Social Science, 26(2), 89 - 97. Doi: https://doi.org/10.1080/097 18923.2011.11892885 